\title{
A catastrophic-cum-restorative queuing system with correlated batch arrivals and variable capacity
}

\author{
Rakesh Kumar \\ School of Mathematics \\ Shri Mata Vaishno Devi University, Katra \\ Sub - Post Office, SMVD University Campus-182320 (J\&K) India
}

\begin{abstract}
In this paper, we study a catastrophic-cum-restorative queuing system with correlated batch arrivals and service in batches of variable sizes. We perform the transient analysis of the queuing model. We obtain the Laplace Transform of the probability generating function of system size. Finally, some particular cases of the model have been derived and discussed.
\end{abstract}

Keywords: Queue length, Catastrophes, Correlated batch arrivals, Broadband services, Variable service capacity, and Restoration.

\section{Introduction}

New Broadband Communication Networks are playing a key role in providing a variety of multimedia services such as voice, video and data etc. The amount of information per unit time generated by these services varies along the connection duration. There are certain periods in which the information rate increases and others in which it decreases or becomes null. As the sources providing such services are not synchronized several cells may arrive at the same slot. Thus, they (cells) arrive in batches of variable size. Due to the bursty nature of the cell traffic generated by broadband services the arrival process in New Broadband Communication Networks is correlated in nature [1]. TCP is the most commonly used transport protocol for Internet broadband services. Because it is a connection-based protocol, TCP is able to guarantee that each data packet transmitted from a server reaches its intended destination. It transmits cells in batches [2].

Further, the arrival of infected cells (viruses) and noise bursts etc. may annihilate all the cells in the buffer of the server (computer) and leave it momentarily inactivated until the new cell arrival occurs. Such infected cells may be modeled by catastrophes. The notion of catastrophes occurring at random, leading to annihilation of all the customers there and the momentary inactivation of service facility until a new arrival of a customer is not uncommon in many practical problems. X. Chao studied a queuing network model with catastrophes [3]. A. DiCrescenzo et al. [4] studied an $\mathrm{M} / \mathrm{M} / 1$ queue with catastrophes and derived its heavy traffic approximation. Recently, Jain and Kumar $[5,6,7]$ obtained the transient solution of some catastrophic queuing systems with correlated input.

The concept of catastrophe has tremendous applications in a wide variety of areas particularly in computer-communication, biosciences, population studies and industries etc. It is based on the assumption that with the occurrence of 
catastrophe, all the customers in the system are destroyed and simultaneously the system becomes ready to accept new customers. However a system will always require some sort of time to function in a normal way if it suffers from catastrophe, which is taken as restoration time. Thus, it would be more practicable if we model the restoration time required by a system which is suffering from catastrophe. In the present example, with the occurrence of catastrophe all the cells in the buffer of the server are destroyed immediately. But the server can work properly after it is free from the viruses and noise bursts. Thus, some short of recovery / restoration time is needed. To this end, the concept of restoration time is introduced in which no arrival is allowed to occur.

In this paper, we incorporate the effect of catastrophes and restoration in the correlated batch arrival queue with variable service capacity which may arise in New Broadband Communication Networks. We consider a single server catastrophic-cum-restorative queuing system with correlated batch input and variable capacity. We derive the transient solution of the model under investigation.

This paper has been organized as follows: In section 2, we formulate the queuing model. In section 3, we obtain the transient solution of the model. The paper is concluded in section 4 .

\section{Queuing Model}

The queuing model under investigation is based on the following assumptions:

(1) The customers arrive at a service facility in batches, the size of the batch being a random variable with Prob. (size of the batch is $\mathrm{j}$ ) $=\mathrm{c}_{\mathrm{j}, \mathrm{j}} \mathrm{j}=1,2,3, \ldots \ldots$... and $\sum_{j=1}^{\infty} c_{j}=1$

(2) The arrival of a batch can occur only at the transition marks $t_{0}, t_{1}, t_{2} \ldots$ where $\theta_{r}=t_{r}-t_{r-1} ; r=1,2,3, \ldots \ldots \ldots$ are random variables with $P\left[\theta_{r} \leq x\right]=1-\exp (-\lambda x) ; \lambda>0, r=1,2, \ldots \ldots \ldots \ldots \ldots$

The arrival and no arrival of a batch at two consecutive transition marks $t_{r-1}, t_{r} ; r=1,2,3, \ldots \ldots .$. are governed by the following transition probability matrix:

To $t_{r}$

arrival of a batch

From $t_{r-1}$

arrival of a batch

no arrival of a batch

Thus, the arrivals of batches at two consecutive transition marks are correlated. 
(3) The capacity of the service channel is determined at the beginning of each service and is a random variable with

Prob. [capacity is j] $=\mathrm{a}_{\mathrm{j}}, \mathrm{j}=0,1,2,3, \ldots \ldots \ldots, \mathrm{M}$ and $\sum_{j=0}^{M} a_{j}=1$.

(4) The queue disciplined is first-come-first-served, so that

(i) if the capacity is ( $j>0)$ and the queue length (the number of units waiting excluding those being served) is $n(>0)$, then a batch of $\min (n, j)$ units is taken for service;

(ii) if at any instant the capacity of the service channel is j $(>0)$ and the queue length is zero, then the service channel will remain idle and the service will start immediately on an arrival of a batch.

(5) The service time distribution is exponential with parameter $\mu$.

(6) When the system is not empty, the catastrophes occur at the service facility according to a Poisson process with rate $\xi$. The catastrophes annihilate all the customers in the system instantaneously.

(7) The restoration times are independently, identically and exponentially distributed with parameter $\eta$.

(8) The stochastic processes namely (i) the distribution of inter-transition times, (ii) the capacity distribution (iii) distribution of the size of arrival of a batch and (iv) the distribution of catastrophes and (v) the distribution of restoration times are independent of each other.

(9) Let the time be reckoned from the instant when the service channel is idle and a transition with no arrival has just occurred, so that

$Q_{0,0}(0)=1$

Define,

$P_{n, 0}(t)=$ the probability that at time $t$, the queue length (the number of customers waiting excluding those being served) is equal to $n$, the service channel is not idle and no arrival of a batch has occurred at the previous transition mark.

$P_{n, 1}(t)=$ the probability that at time $t$, the queue length is equal to $n$, the service channel is not idle and an arrival of a batch has occurred at the previous transition mark.

$Q_{0,0}(t)=$ the probability that at time $t$, the queue length is equal to 0 without the occurrence of catastrophe, the service channel is idle and no arrival of a batch has occurred at the previous transition mark.

$\mathrm{C}_{0,0}(\mathrm{t})=$ the probability that at time $\mathrm{t}$, the queue length is equal to 0 with the occurrence of catastrophe, the service channel is idle and no arrival of a batch has occurred at the previous transition mark.

$Q_{0,1}(t)=$ the probability that at time $t$, the queue length is equal to 0 without the occurrence of catastrophe, the service channel is idle and an arrival of a batch has occurred at the previous transition mark. 
$\mathrm{C}_{0,1}(\mathrm{t})=$ the probability that at time $\mathrm{t}$, the queue length is equal to 0 with the occurrence of catastrophe, the service channel is idle and an arrival of a batch has occurred at the previous transition mark.

$R_{n}(t)=$ the probability that at time $t$, the queue length is equal to $n$.

\section{Transient solution of the Model}

The equations governing the model are: -

$R_{n}(t)=P_{n, 0}(t)+P_{n, 1}(t) ; n=1,2,3 \ldots .$.

$\mathrm{R}_{0}(\mathrm{t})=\mathrm{P}_{0,0}(\mathrm{t})+\mathrm{P}_{0,1}(\mathrm{t})+\mathrm{Q}_{0,0}(\mathrm{t})+\mathrm{Q}_{0,1}(\mathrm{t})$

$\frac{d}{d t} Q_{0,0}(t)=-\lambda Q_{0,0}(t)+\mu\left(1-a_{0}\right) P_{0,0}(t)+\lambda\left[p_{00} Q_{0,0}(t)+p_{10} Q_{0,1}(t)\right]+\eta C_{0,0}(t)$

$\frac{\mathrm{d}}{\mathrm{dt}} \mathrm{C}_{0,0}(\mathrm{t})=-\eta C_{0,0}(t)+\xi\left[\sum_{n=0}^{\infty} P_{n, 0}(t)\right]$

$\frac{d}{d t} Q_{0,1}(t)=-\lambda Q_{0,1}(t)+\mu\left(1-a_{0}\right) P_{0,1}(t)+\eta C_{0,1}(t)$

$\frac{\mathrm{d}}{\mathrm{dt}} \mathrm{C}_{0,1}(\mathrm{t})=-\eta C_{0,1}(t)+\xi\left[\sum_{n=0}^{n=\infty} P_{n, 1}(t)\right]$

$\frac{d}{d t} P_{0,0}(t)=-(\lambda+\mu+\xi) P_{0,0}(t)+\mu a_{0} P_{0,0}(t)+\sum_{j=1}^{M} \sum_{r=1}^{j} \mu a_{j} P_{r, 0}(t)+\lambda\left[p_{00} P_{0,0}(t)+p_{10} P_{0,1}(t)\right]$

$\frac{d}{d t} P_{n, 0}(t)=-(\lambda+\mu+\xi) P_{n, 0}(t)+\sum_{j=1}^{M} \mu a_{j} P_{n+j, 0}(t)+\lambda\left[p_{00} P_{n, 0}(t)+p_{10} P_{n, 1}(t)\right] ; \mathrm{n}=1,2, .$.

$\frac{d}{d t} P_{0,1}(t)=-(\lambda+\mu+\xi) P_{0,1}(t)+\mu a_{0} P_{0,1}(t)+\sum_{j=1}^{M} \sum_{r=1}^{j} \mu a_{j} P_{r, 1}(t)+\lambda\left[p_{01} Q_{0,0}(t)+p_{11} Q_{0,1}(t)\right]$

$\frac{d}{d t} P_{n, 1}(t)=-(\lambda+\mu+\xi) P_{n, 1}(t)+\sum_{j=1}^{M} \mu a_{j} P_{n+j, 1}(t)+\lambda \sum_{j=1}^{n} c_{j}\left[p_{01} P_{n-j, 0}(t)+p_{11} P_{n-j, 1}(t)\right]$

$; n=1,2, \ldots$

Define the Laplace transform of $f(t)$ by

$f^{*}(s)=\int_{0}^{\infty} e^{-s t} f(t) d t$

Taking L.T.'s of (1) - (10), we have

$R^{*} n(s)=P^{*}{ }_{n, 0}(s)+P^{*}{ }_{n, 1}(s) \quad ; n=1,2,3, \ldots \ldots .$.

$\mathrm{R}_{0}^{*}(\mathrm{~s})=\mathrm{P}_{0,0}^{*}(\mathrm{~s})+\mathrm{P}_{0,1}^{*}(\mathrm{~s})+\mathrm{Q}_{0,0}^{*}(\mathrm{~s})+\mathrm{Q}_{0,1}^{*}(\mathrm{~s})$

$(s+\lambda) Q_{0,0}^{*}(s)-1=\mu\left(1-a_{0}\right) P_{0,0}^{*}(s)+\lambda\left[p_{00} Q_{0,0}^{*}(s)+p_{10} Q_{0,1}^{*}(s)\right]+\eta C_{0,0}^{*}(s)$

$(s+\eta) C_{0,0}^{*}(s)=\xi\left[\sum_{n=0}^{n=\infty} P_{n, o}^{*}(s)\right]$

$(s+\lambda) Q_{0,1}^{*}(s)=\mu\left(1-a_{0}\right) P_{0,1}^{*}(s)+\eta C_{0,1}^{*}(s)$

$(s+\eta) C_{0,1}^{*}(s)=\xi\left[\sum_{n=0}^{n=\infty} P_{n, 1}^{*}(s)\right]$

$(s+\lambda+\mu+\xi) P_{0,0}^{*}(s)=\mu a_{0} P_{0,0}^{*}(s)+\sum_{j=1}^{M} \sum_{r=1}^{j} \mu a_{j} P_{r, 0}^{*}(s)+\lambda\left[p_{00} P_{0,0}^{*}(s)+p_{10} P_{0,1}^{*}(s)\right]$ 


$$
\begin{gathered}
(s+\lambda+\mu+\xi) P_{n, 0}^{*}(s)=\sum_{j=1}^{M} \mu a_{j} P^{*}{ }_{n+j, 0}(s)+\lambda\left[p_{00} P^{*}{ }_{n, 0}(s)+p_{10} P_{n, 1}^{*}(s)\right] ; \mathrm{n}=1,2, \ldots \\
(s+\lambda+\mu+\xi) P^{*}{ }_{0,1}(s)=\mu a_{0} P^{*}{ }_{0,1}(s)+\sum_{j=1}^{M} \sum_{r=1}^{j} \mu a_{j} P_{r, 1}^{*}(s)+\lambda\left[p_{01} Q_{0,0}^{*}(s)+p_{11} Q^{*}{ }_{0,1}(s)\right] \\
(s+\lambda+\mu+\xi) P_{n, 1}^{*}(s)+\sum_{j=1}^{M} \mu a_{j} P^{*}{ }_{n+j, 1}(s)+\lambda \sum_{j=1}^{n} c_{j}\left[p_{01} P_{n-j, 0}^{*}(s)+p_{11} P_{n-j, 1}^{*}(s)\right] ; \\
\mathrm{n}=1,2, \ldots
\end{gathered}
$$

Let us define probability generating functions by

$P^{*}{ }_{i}(s, \alpha)=\sum_{n=0}^{\infty} P^{*}{ }_{n, i}(s) \alpha^{n} ; \mathrm{i}=0,1$

$R^{*}(s, \alpha)=\sum_{n=0}^{\infty} R_{n}^{*}(s) \alpha^{n}$

Multiplying (18) and (19) by appropriate powers of $\alpha$ and adding, we have

$\left(h-\lambda p_{00} \alpha^{M}\right) P^{*}{ }_{0}(s, \alpha)-\lambda p_{10} \alpha^{M} P^{*}{ }_{1}(s, \alpha)=\sum_{r=0}^{M-1} g_{r} P_{r, 0}^{*}(s)-\mu\left(1-a_{0}\right) \alpha^{M} P_{0,0}^{*}(s)$

where $g_{r}=\sum_{j=r+1}^{M} \mu a_{j}\left(\alpha^{M}-\alpha^{M+r-j}\right)$

and $h=(s+\lambda+\mu+\xi) \alpha^{M}-\sum_{j=0}^{M} \mu a_{j} \alpha^{M-j}$

Similarly, (20) and (21) give

$\left(h-\lambda p_{11} \alpha^{M} C(\alpha)\right) P^{*}{ }_{1}(s, \alpha)-\lambda p_{01} \alpha^{M} C(\alpha) P^{*}{ }_{0}(s, \alpha)=\sum_{r=0}^{M-1} g_{r} P^{*}{ }_{r, 1}(s)-\mu\left(1-a_{0}\right) \alpha^{M} P_{0,1}^{*}(s)+$

$\lambda \alpha^{M}\left[p_{01} Q^{*}{ }_{0,0}(s)+p_{11} Q_{0,1}(s)\right]$

and from (12) and (13) we have

$\mathrm{R}^{*}(\mathrm{~s}, \alpha)=\mathrm{P}_{0}^{*}(\mathrm{~s}, \alpha)+\mathrm{P}_{1}^{*}(\mathrm{~s}, \alpha)+\mathrm{Q}_{0,0}^{*}(\mathrm{~s})+\mathrm{Q}_{0,1}^{*}(\mathrm{~s})$

Solving (24) and (27) simultaneously, we have

$\mathrm{P}_{\mathrm{i}}^{*}(\mathrm{~s}, \alpha)=\frac{N_{i}(\alpha)}{D(\alpha)} \quad ; \mathrm{i}=0,1$

Where

$$
\begin{aligned}
N_{0}(\alpha)=\lambda & \left.p_{10} \alpha^{M}\left[\sum_{r=0}^{M-1} g_{r} P_{r, 1}^{*}(s)-\mu\left(1-a_{0}\right) \alpha^{M} P_{0,1}^{*}(s)+\lambda \alpha^{M}\left[p_{0,1} Q_{0,0}^{*}(s)+p_{11} Q^{*}{ }_{0,1}(s)\right]\right]\right]+ \\
& \left(h-\lambda p_{11} \alpha^{M} C(\alpha)\right)\left[\sum_{r=0}^{M-1} g_{r} P_{r, 0}^{*}(s)-\mu\left(1-a_{0}\right) \alpha^{M} P^{*}{ }_{0,0}(s)\right] \\
N_{1}(\alpha)=\lambda & p_{01} \alpha^{M} C(\alpha)\left[\sum_{r=0}^{M-1} g_{r} P_{r, 0}^{*}(s)-\mu\left(1-a_{0}\right) \alpha^{M} P_{0,0}^{*}(s)\right]+\left(h-\lambda p_{00} \alpha^{M}\right) \\
& {\left[\sum_{r=0}^{M-1} g_{r} P_{r, 1}^{*}(s)-\mu\left(1-a_{0}\right) \alpha^{M} P^{*}{ }_{0,1}(s)+\lambda \alpha^{m}\left[p_{0,1} Q^{*}{ }_{0,0}(s)+p_{11} Q^{*}{ }_{0,1}(s)\right]\right] }
\end{aligned}
$$


and

$D(\alpha)=\left[h-\lambda p_{00} \alpha^{M}\right]\left[h-\lambda p_{11} C(\alpha) \alpha^{M}\right]-\lambda^{2} p_{01} p_{10} \alpha^{2 M} C(\alpha)$

Combining (24), (25) and (26) we get

$\mathrm{R}^{*}(\mathrm{~s}, \alpha)=\mathrm{Q}_{0,0}^{*}(\mathrm{~s})+\mathrm{Q}_{0,1}^{*}(\mathrm{~s})+\frac{N(\alpha)}{D(\alpha)}$

Where

$\mathrm{N}(\alpha)=\mathrm{N}_{0}(\alpha)+\mathrm{N}_{1}(\alpha)$

$N(\alpha)=\left[h+\lambda\left(p_{10}-p_{00}\right) \alpha^{M}\right]\left[\sum_{r=0}^{M-1} g_{r} P_{r, 1}^{*}(s)-\mu\left(1-a_{0}\right) \alpha^{M} P_{0,1}^{*}(s)+\lambda \alpha^{M}\left(p_{01} Q_{0,0}^{*}(s)+p_{11} Q^{*}{ }_{0,1}(s)\right)\right]+$

$\left[h+\lambda\left(p_{01}-p_{11}\right) \alpha^{M} C(\alpha)\right]\left[\sum_{r=0}^{M-1} g_{r} P_{r, 0}^{*}(s)-\mu\left(1-a_{0}\right) \alpha^{M} P_{0,0}^{*}(s)\right]$

From (22) we have for $\alpha=1$

$$
P^{*}(s, 1)=\sum_{n=0}^{\infty} P^{*}{ }_{n, 0}(s) \text { and } P^{*}{ }_{1}(s, 1)=\sum_{n=0}^{\infty} P^{*}{ }_{n, 1}(s)
$$

Substituting for $\alpha=1$ in (29) and using (33), we get

$$
\sum_{n=0}^{\infty} P_{n, 0}^{*}(s)=\frac{N_{0}(1)}{D(1)} \quad \text { and } \quad \sum_{n=0}^{\infty} P_{n, 1}^{*}(s)=\frac{N_{1}(1)}{D(1)}
$$

Also from (15) and (17), we have

$$
C^{*}{ }_{0,0}(s)=\left(\frac{\xi}{s+\eta}\right)\left[\sum_{n=0}^{n=\infty} P_{n, o}^{*}(s)\right]
$$

and

$$
C_{0,1}^{*}(s)=\left(\frac{\xi}{s+\eta}\right)\left[\sum_{n=0}^{\infty} P_{n, 1}^{*}(s)\right]
$$

Thus, substituting the values of $C^{*}{ }_{0,0}(s)$ and $C_{0,1}^{*}(s)$ in (14) and (16) we get two equations in four unknowns.

By Rouche's theorem the denominator $D(\alpha)$ in (31) has $2 \mathrm{M}$ zeros inside the unit circle $|\alpha|=1$. Since, $R^{*}(s, \alpha)$ is a finite quantity, these zeros must vanish the numerator $N(\alpha)$ giving rise to a set of $2 \mathrm{M}$ equations. Solving these $2 \mathrm{M}$ equations together with (14) and (16) one can determine all the $2 \mathrm{M}+2$ unknowns occurring in the numerator $\mathrm{N}(\alpha)$. Hence, $R^{*}(s, \alpha)$ can be completely determined. 


\section{Particular cases}

(1) When $\xi=0$ and $\eta=\infty$ (i.e. neither catastrophe nor restoration occurs) the model reduces to a correlated batch arrival queuing system with variable capacity having the Laplace transform of probability generating function of system size as given below

$$
\mathrm{R}^{*}(\mathrm{~s}, \alpha)=\mathrm{Q}_{0,0}^{*}(\mathrm{~s})+\mathrm{Q}_{0,1}^{*}(\mathrm{~s})+\frac{N(\alpha)}{D(\alpha)}
$$

$$
\begin{gathered}
N(\alpha)=\left[h+\lambda\left(p_{10}-p_{00}\right) \alpha^{M}\left[\sum_{r=0}^{M-1} g_{r} P_{r, 1}^{*}(s)-\mu\left(1-a_{0}\right) \alpha^{M} P_{0,1}^{*}(s)+\lambda \alpha^{M}\left(p_{01} Q_{0,0}^{*}(s)+p_{11} Q_{0,1}^{*}(s)\right)\right]+\right. \\
{\left[h+\lambda\left(p_{01}-p_{11}\right) \alpha^{M} C(\alpha)\right]\left[\sum_{r=0}^{M-1} g_{r} P_{r, 0}^{*}(s)-\mu\left(1-a_{0}\right) \alpha^{M} P^{*}{ }_{0,0}(s)\right]} \\
\mathrm{D}(\alpha)=\left[\mathrm{h}-\lambda \mathrm{p}_{00} \alpha^{\mathrm{M}}\right]\left[\mathrm{h}-\lambda \mathrm{p}_{11} \mathrm{C}(\alpha) \alpha^{\mathrm{M}}\right]-\lambda^{2} \mathrm{p}_{01} \mathrm{p}_{10} \alpha^{2 \mathrm{M}} \mathrm{C}(\alpha) \\
\text { Where } h=(s+\lambda+\mu) \alpha^{M}-\sum_{j=0}^{M} \mu a_{j} \alpha^{M-j} \\
C^{*}{ }_{0,0}(s)=C^{*}{ }_{0,1}(s)=0
\end{gathered}
$$

(2) When the arrivals occur one by one (i.e. $C(\alpha)=\alpha$ ) and $\xi=0, \eta=\infty$ the model reduces to that studied by Mohan and Murari [8] having the Laplace transform of probability generating function of system size as given below:

$$
\begin{aligned}
& R^{*}(s, \alpha)=Q_{0,0}^{*}(s)+Q_{0,1}^{*}(s)+\frac{N(\alpha)}{D(\alpha)} \\
& N(\alpha)=\left[h+\lambda\left(p_{10}-p_{00)} \alpha^{M}\right]\left[\sum_{r=0}^{M-1} g_{r} P_{r, 1}^{*}(s)-\mu\left(1-a_{0}\right) \alpha^{M} P_{0,1}^{*}(s)+\lambda \alpha^{M}\left(p_{01} Q^{*}{ }_{0,0}(s)+p_{11} Q^{*}{ }_{0,1}(s)\right)\right]+\right. \\
& {\left[h+\lambda\left(p_{01}-p_{11}\right) \alpha^{M+1}\right]\left[\sum_{r=0}^{M-1} g_{r} P_{r, 0}^{*}(s)-\mu\left(1-a_{0}\right) \alpha^{M} P^{*}{ }_{0,0}(s)\right]} \\
& D(\alpha)=\left(h-\lambda p_{00} \alpha^{M}\right)\left(h-\lambda p_{11} \alpha^{M+1}\right)-\lambda^{2} p_{01} p_{10} \alpha^{2 M+1} \\
& \text { Where } h=\alpha(s+\lambda+\mu+\xi)-\mu
\end{aligned}
$$

\section{Conclusion}

A catastrophic queuing model with correlated batch arrivals and variable capacity is studied with reference to its application in new Broadband Communication networks. Some queuing models have been derived as particular cases.

\section{References}

1. A. DiCrescenzo, V. Giorno and L.M. Ricciardi, "On the M/M/1 queue with catastrophes and its continuous approximation", Queueing Systems 43, 329-347 (2003).

2. C. Mohan and K. Murari, "Time dependent solution of correlated queuing problem with variable capacity, Metrika, 19,209-215 (1972). 
3. J. Andrade Parra, "Statistical parameters to describe cell traffic generated by broadband services", Communicaciones de Telefonica, No.8, December, 1993.

4. Lior Storfer, "Enhancing Cable Modem TCP Performance" White paper, Cable Digital News ${ }^{\mathrm{TM}}$, Broadband Communications and Entertainment Business Insight.

5. N.K. Jain and Rakesh Kumar, "A catastrophic queuing system with correlated batch arrivals and variable service capacity", Bulletin of Pure and Applied Sciences, Vol. 25E, No.1, 97-104 (2006).

6. N.K. Jain and Rakesh Kumar, "A catastrophic queuing model with correlated input for the cell traffic generated by new broadband services", Indian Journal of Mathematics and Mathematical Sciences, Vol.1, No.2, 99-108 (2005).

7. N.K. Jain and Rakesh Kumar, "Transient solution of a correlated queuing Problem with variable capacity and catastrophes", International Journal of Information and Management Sciences, Vol.16, No.4, 39-47 (2005).

8. X. Chao, "A queueing network model with catastrophes and product form solution", O.R. Letters 18, 75-79 (1995). 